\title{
INFESTACION DOMESTICA POR TRLATOMA INFESTANS Y PREVALENCIA DE SEROPOSITIVOS AL TRYPANOSOMA CRUZI EN UN AREA RURAL DEL NORDESTE ARGENTINO
}

\author{
M. Esther Bar, E.B. Oscherov, M.P. Damborsky, B.M. Alvarez, G. Mizdraji, \\ G. Avalos y E. Porcel
}

\begin{abstract}
Se lletó a cabo un estudio epidemiologico para obtener un panorama general de la transmisión doméstica del Trypanosoma cruzi en äreas rurales del Departamento San Miguel, Comientes, Argentina. Se investigaron 100 viniendas precarias, 50,0\% de las mismas resultó infestado por Triatoma infestans $y 1,0 \%$ por $\mathrm{T}$. sordida, citándose por primera vez para la prozincia de Comientes la colonización domiciliaria de esta especie. El 23,1\% de T. infestans estaba infectado por T. cruzi. La seroprevalencia de 388 pacientes analizados por bemaglutinación indirecta e inmunofluorescencia indirecta fue 23,4\%, destacändose el alto indice (12,9\%) constatado en menores de 10 años. Los porcentajes de seropositividad aumentaron con la edad, alcanzándose 50,0\% de infectados entre los 31-40 años. La infestación doméstica por $T$. infestans, la pretalencia bumana de seropositivos al $\mathrm{T}$. cruzi y las condiciones de precariedad en que se desenuelte la vida de los pobladores revelan la tigencia de la endemia chagásica en el área estudiada.
\end{abstract}

Palabras-claves: Triatominos. Infestación. T. cruzi. Seropretalencia. Corrientes.

La infección tripanosómica depende fundamentalmente de la convergencia, en determinado tiempo y lugar, del agente etiológico, del insecto vector, del hospedador animal o humano y del susceptible, todo dentro de un contexto geográfico favorable ${ }^{8}$.

Los vectores del T. cruzi están distribuídos en todo el continente americano, entre las latitudes $42^{\circ} \mathrm{N}$ y $46^{\circ} \mathrm{S}$, estimándose que la prevalencia en Argentina es de aproximadamente 2.640.000 seropositivos ${ }^{19}$.

La infestación triatomínica en la provincia de Corrientes ofrece características tales como: altos índices de colonización domiciliaria por T. infestans en el Dpto. San Luis del Palmar ${ }^{3}$, una infestación menor en el Dpto. Empedrado, hallazgos aislados atribuidos a la dispersión pasiva en áreas rurales del Dpto. Mercedes ${ }^{2}$ y presencia de ejemplares muertos, exuvias y rastros de materia fecal, en la ciudad de Corrientes, (M.E. Bar e cols: datos no publicados).

Trabajo financiado por la Secretaría de Ciencia y Técnica de la Universidad Nacional del Nordeste.

Endereço para comespondência: $\mathrm{Dr}^{\mathrm{a}}$ María Esther Bar. Cátedra de Artrópodos. Facultad de Ciencias Exactas y Naturales y Agrimensura. UNNE. 9 de Juhio 1449. 3400. Corrientes.Argentina. Fax: 0783-23968.

Recebido para publicação em 13/10/95
Los valores de seroprevalencia de anticuerpos contra el $T$. cruzi registrados en la provincia de Corrientes, varían desde 4,9\% en pobladores (urbanos y rurales) del Dpto. Monte Caseros $^{17}, 22,3 \%$ en el Dpto. San Luis del Palmar $^{3}, 46,0 \%$ en el Dpto. Ituzaingó ${ }^{\circ}$, a $63,3 \%$ en soldados de la ciudad de Corrientes.

Esta realidad configura una situación epidemiológica heterogénea y a su vez diferente a la de otras regiones altamente endémicas de Argentina, como Santiago del Estero ${ }^{2+}$ y Córdoba ${ }^{18}$.

En 1982 se inició un proyecto con el propósito de obtener las características epidemiológicas de la enfermedad de Chagas en la provincia de Corrientes, ya que los datos de que se disponían eran escasos y no actualizados. La presente investigación, continuación del citado proyecto, tiene como objetivo específico conocer la infestación domiciliaria por triatominos, el porcentaje de infección de $T$. infestans por $T$. cruzi y la prevalencia humana de seropositivos al $T$. cruzi en el Dpto. San Miguel.

\section{MATERIALES Y METODOS}

Area de estudio. El Dpto. San Miguel se halla ubicado en la región Nordeste de la provincia de Corrientes, Argentina $\left(28^{\circ} \mathrm{S}\right.$; 
Bar ME, Oscherov EB, Damborsky MP, Alvarez BM, Mizdraji G, Avalos G, Porcel E. Infestacion domestica por Triatoma infestans y prevalencia de seropositivos al Trypanosoma cruzi en un area rural del nordeste argentino. Revista da Sociedade Brasileira de Medicina Tropical 29:549-555. nov-dez, 1996.

$57^{\circ} 34^{\prime} \mathrm{W}$ ) (Figura 1). Tiene una superficie de $3.018 \mathrm{~km}^{2}$, con 9.169 habitantes, concentrándose el $52,0 \%$ de la población en la zona rural (Censo Nacional de Población y Vivienda 1991). El clima es subtropical', con precipitaciones anuales de $1.300 \mathrm{~mm}$, distribuidas durante todo el año. La temperatura media anual es de $21^{\circ} \mathrm{C}$ y la humedad relativa de $75 \% \%^{21}$. El relieve es llano con esteros y lagunas; este paisaje palustre alterna con lomadas que sirven de asiento al hombre, los suelos son aptos para el cultivo, lo que asociado a la explotación ganadera constituyen los principales medios de subsistencia de la población ${ }^{7}$. Existen además extensas áreas de bosques naturales así como forestadas de Pinus sp y Eucaliptus $\mathrm{sp}^{11}$.
Actividades de campo y de laboratorio. El muestreo entomoepidemiológico fue llevado a cabo desde abril de 1991 hasta diciembre de 1993 en las colonias* San Antonio y Caimán, las cuales comprenden el $28,0 \%$ de las viviendas rurales del Dpto. San Miguel.

Al no tener referencias previas acerca de la prevalencia de infección por $T$. cruzi, para estimar el tamaño aproximado de la muestra se seleccionó un valor del $50,0 \%$ con un nivel de confianza del $95,0 \%$ y una precisión del $5,0 \% \%^{22}$. El tamaño de la muestra resultó igual a 384 .

Se seleccionaron 43 casas en Colonia San Antonio y 57 en colonia Caimán, las cuales no constituian un caserio sino que estaban separadas por distancias que oscilaban entre 30 y 600 metros.

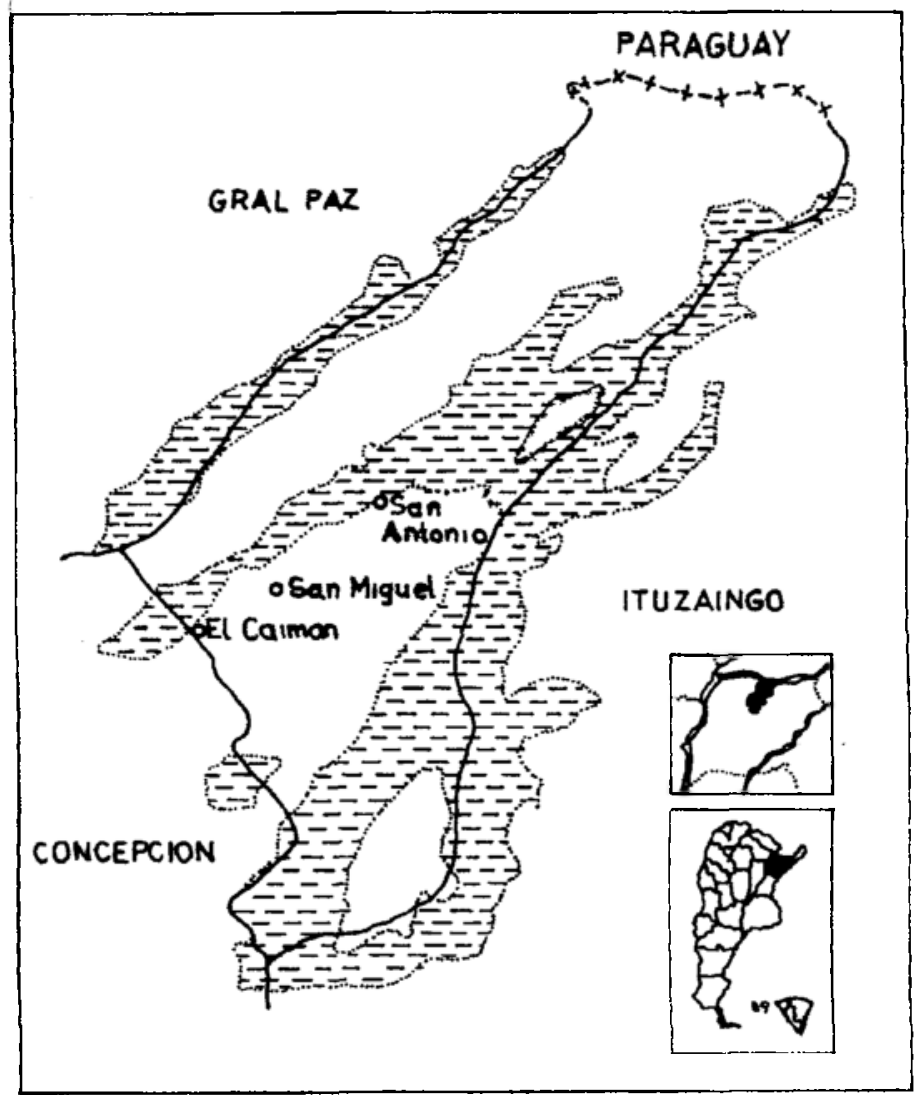

Figura 1 - Localización de las colonias estudiadas en el Dpto. San Miguel, Corrientes.

\footnotetext{
* comunidad rural dedicada a determinada actividad agrícola.
} 
Bar ME, Oscherov EB, Damborsky MP, Altarez BM, Mizdraji G, Avalos G, Porcel E. Infestacion domestica por Triatoma infestans y prevalencia de seropositivos al Trypanosoma cruzi en un area rural del nordeste argentino. Revista da Sociedade Brasileira de Medicina Tropical 29:549-555, nov-dez, 1996.

La búsqueda de los triatominos fue realizada por un grupo de cuatro personas durante una hora/hombre ${ }^{23}$, en diferentes sectores del domicilio, los cuales fueron rociados con neopynamin al $0,2 \%$, provocando que las chinches abandonaran sus refugios. Los insectos colectados fueron conservados en frascos rotulados y en laboratorio fueron clasificados por especie, estructura de edad y $\mathrm{sexo}^{1+}$. El contenido rectal de los triatominos fue diluído en solución de cloruro de sodio al $0,85 \%$ y observado al microscopio con 400x para confirmar la presencia del T. cmizi.

El peridomicilio fue analizado utilizándose la misma metodología que en el domicilio. También fueron disecados ecótopos silvestres consistentes en palmeras (Butia yatay).

Mediante encuestas a los jefes de familia se obtuvo información acerca de las características de la vivienda, presencia de animales domésticos. uso de insecticidas, número de personas residentes, edad, sexo, actividad laboral. migración y nivel de escolaridad.

Para la evaluación serológica se extrajo sangre a los moradores por punción venosa. Las muestras de suero fueron conservadas en freezer $\left(-20^{\circ} \mathrm{C}\right)$ hasta su procesamiento. Las técnicas inmunológicas utilizadas para el diagnóstico serológico confirmatorio fueron la hemaglutinación indirecta ${ }^{13}$ e inmunofluorescencia indirecta ${ }^{1}$ desarrolladas en forma cuantitativa. Se consideraron positivos aquellos pacientes cuyos sueros presentaron títulos $\geq 1 / 32$ en ambas pruebas.

En el tratamiento estadístico de los datos se utilizó la prueba del $\chi^{2}$. La relación entre la densidad de triatominos y la de $T$. infestans infectada con T. cruzi fue analizada por medio de los tests de Kruskal- Wallis (H) y MannWhitney $(\mathrm{U})^{20}$, a un nivel de significación del $5,0 \%$, ya que los datos no respondian a una distribución normal.

\section{RESULTADOS}

Características de la vivienda. El 55,0\% de las 100 viviendas investigadas tenían 10 o más años de antiguedad. Los materiales de construcción predominantes fueron: paredes de "estanteo"* (Figura 2) y sus combinaciones

\footnotetext{
* material resultante de la mezcla de tierra, arena y agua,
} soportado por troncos de especies vegetales autóctonas.
$(70,0 \%)$. El "estanteo" constituía el material único de las paredes en el 34,0\% de las viviendas y estaba acompañado por caña (Guadua sp.) en un 23,0\%, y por paja (Panicum prionitis) u otros materiales en un 13,0\%. Las paredes presentaban externamente una cobertura de caña y paja, ofreciendo a los triatominos mayores posibilidades de refugio. El $91,0 \%$ de los techos eran de paja, con soportes de caña, los restantes eran de chapas de zinc y/o cartón. El 93,0\% de los pisos eran de tierra. En el interior de un elevado número de viviendas $(n=84)$ dormian animales domésticos en una proporción de: perros $(71,0 \%)$, aves $(65,0 \%)$, gatos $(38,0 \%)$ y otros $(5,0 \%)$

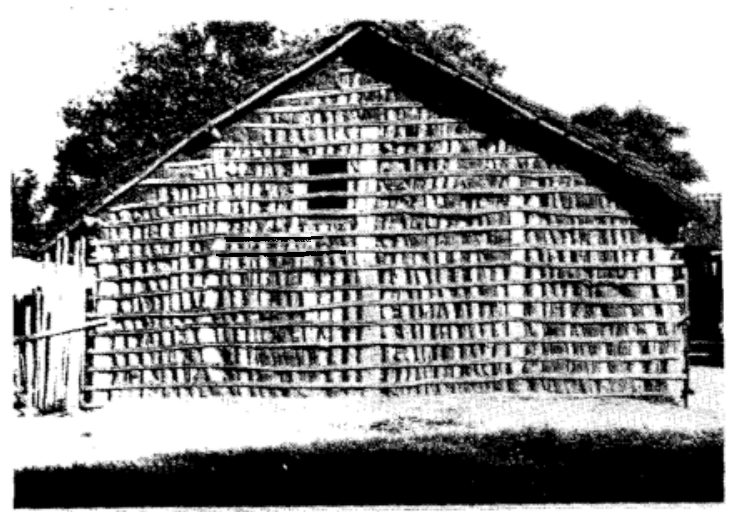

Figura 2 - Vivienda precaria del Dpto. San Miguel, Corrientes

Infestación e infección triatominica. El $50,0 \%$ de las viviendas estaban colonizadas por $T$. infestans y el $1,0 \%$ por $T$. sordida. Se capturaron 355 ejemplares de $T$. infestans (Tabla 1), en su mayoría ninfas $(61,9 \%)$. Ocho huevos estaban parasitados por el microhymenóptero Telenomus fariai.

Con referencia a la presencia domiciliaria de $T$. sordida se destaca que es el primer hallazgo de esta especie colonizando la vivienda humana que se cita para la provincia de Corrientes. La población total detectada fue de 73 individuos, la mayoría eran huevos y adultos. Los ejemplares vivos estaban refugiados en la paja del techo, en una de las paredes de junco (Scirpus californicus) y en la cama del morador.

En las viviendas de la colonia San Antonio (25/43) la presencia de triatominos fue 
Bar ME, Oscherou EB, Damborsky MP, Alwarez BM, Mizdraji G, Alalos G, Porcel E. Infestacion domestica por Triatoma infestans $y$ prevalencia de seropositivos al Trypanosoma cruzi en un area rural del nordeste argentino. Revista da Sociedade Brasileira de Medicina Tropical 29:549-555, nov-dez, 1996.

Tabla 1 - Población total de Triatoma infestans y T. sordida capturados en el Dpto. San Miguel. Corrientes, Argentina. 1993

\begin{tabular}{|c|c|c|c|c|c|c|c|c|c|}
\hline \multirow[b]{2}{*}{ Especie } & \multicolumn{9}{|c|}{ Estados de desarrollo } \\
\hline & huevos & N1 & $\mathrm{N} 2$ & $\mathrm{~N} 3$ & N4 & N5 & machos & hembras & total \\
\hline \multicolumn{10}{|l|}{ T. infestans } \\
\hline Colectados vivos & 78 & 18 & 68 & 48 & 49 & 37 & 33 & 24 & 355 \\
\hline Colectados muertos & 20 & 8 & 18 & 6 & 5 & 7 & 5 & 7 & 76 \\
\hline Total & 98 & 26 & 86 & 54 & 54 & 44 & 38 & 31 & 431 \\
\hline$\%$ & 22,8 & 6,0 & 20.0 & 12,6 & 12,3 & 10,2 & 8.9 & 7,2 & 100 \\
\hline Colectudos vivos & 34 & - & - & - & 1 & 2 & 4 & 12 & 53 \\
\hline Colectados muertos & 1 & - & - & 3 & - & - & 10 & 6 & 20 \\
\hline Total & 35 & - & - & 3 & 1 & 2 & 14 & 18 & 73 \\
\hline$\%$ & 47.9 & - & - & 4.1 & 1,4 & 2.7 & 19,2 & 24,7 & 100 \\
\hline
\end{tabular}

comprobada en un $58,1 \%$, reduciéndose a $45,6 \%$ en Caimán (26/57), no se observaron diferencias significativas $\left(\boldsymbol{\chi}^{2}=1,54 ; p=0,21\right)$.

En los domicilios que albergaban animales domésticos en su interior se capturaron en promedio, 9 triatominos vivos, valor que descendió a 3,2 en aquellos donde no había animales.

En los primeros años de la década de 1980 el Servicio Nacional de Chagas roció San Antonio y Caimán con Mercaptotian 95\% (Ministerio de Salud Pública: Servicio Provincial de Chagas, comunicación personal, 1995). En el período que abarcó la investigación los Agentes de Salud Rural fumigaron la zona con piretroides. De las viviendas fumigadas $(n=57)$ en el $52,6 \%$ se comprobó infestación doméstica, así como también en el $48,8 \%$ de las no fumigadas $\left(\chi^{2}=\right.$ 0,$14 ; p=0,71$ ).

Se analizaron 59 estructuras peridomiciliarias representadas por corrales de aves (77,9\%), corrales de cerdos $(11,9 \%)$, pilas de troncos y otros $(10,2 \%)$; en ninguna de ellas se constató infestación por triatominos.

El porcentaje de T. infestans infectados por T. crizi fue del 23,1\% (47/203). Los valores de infección por estadios ninfales y adultos fueron: $6 \mathrm{~N}_{2} ; 9 \mathrm{~N}_{3} ; 8 \mathrm{~N}_{4} ; 16 \mathrm{~N}_{5} ; 4$ hembras y 4 machos.

Se verificó que cuando aumentaba la densidad de los triatominos en las viviendas, el número de $T$. infestans infectado por T. cruzi era significativamente mayor que en las viviendas con pocos ejemplares $(\mathrm{U}=12, \mathrm{p}=$ $0.001 ; \mathrm{H}=0,12, \mathrm{p}=0.001$ )

En el hábitat silvestre se disecaron ejemplares de Butia yatay, en las que se capturó T. sordida. (Los resultados sobre ecótopos selváticos serán publicados separadamente ).

Factores socioeconómicos. Del análisis de la encuesta se desprende que: a) el $86,0 \%$ de las viviendas eran habitadas por residentes permanentes, en el 14,0\% restante los pobladores habían migrado temporalmente a zonas de mayor endemicidad chagásica; b) de la población escolar comprendida entre 6 a 14 años, $(n=120)$ el 90,8\% asistían a establecimientos escolares de nivel primario; c) el 74,0\% de los jefes de familia se dedicaban a cultivos de subsistencia en pequeñas parcelas; d) en el $90,0 \%$ de las viviendas los moradores manifestaron que usaban insecticidas.

Muestreo serológico. La población total censada fue de 570 personas, de las cuales 388 $(68,0 \%)$ fueron analizadas serológicamente (Tabla 2). Las edades de los pacientes de ambos sexos, oscilaban entre 2 y 90 años y al menos 1 de los habitantes era reactivo en el $54,0 \%$ de las casas infestadas, 2 en el 26,0\%, 3 en el $12,0 \%$ y de 5 a 8 en el $8,0 \%$ de los restantes domicilios.

La prevalencia general de seropositivos al T. cruzi fue de $23,4 \%$. Se destaca que el $20,0 \%$ de los infectados residentes en viviendas sin triatominos había vivido en alguna oportunidad en áreas de mayor endemicidad.

El grupo etario de 0-10 años presentó un $12,9 \%$ de infectados, valor considerado importante para la zona de estudio, resaltándose que los niños habitaban en mayor proporción, en casas infestadas por triatominos $\left(\chi^{2}=10,65 ; \mathrm{p}=0,0011\right)$. El porcentaje de seropositivos aumentó con la edad, observándose diferencias significativas entre los intervalos analizados $\left(\chi^{2}=24,17\right.$; $\mathrm{GL}=7 ; \mathrm{p}=0,0021$ ); el crecimiento fue rápido y constante hasta los 40 años, el 50,0\% de 
Bar ME, Oscherou EB, Damborsky MP, Alvarez BM. Mizdraji G, Avalos G, Porcel E. Infestacion domestica por Triatoma infestans y prevalencia de seropositivos al Trypanosoma cruzi en un area rural del nordeste argentino. Revista da Sociedade Brasileira de Medicina Tropical 29:549-555, nov-dez, 1996.

Tabla 2 - Distribución por sexo y edad de los serorreactivos al T. cruzi. Dpto. San Miguel. Corrientes, Argentina. 1993.

\begin{tabular}{|c|c|c|c|c|c|c|c|}
\hline \multirow[t]{2}{*}{ Edad } & \multicolumn{3}{|c|}{ Población examinada } & \multicolumn{4}{|c|}{ Seropositivos } \\
\hline & fem & masc & total & fem & masc & total & $\%$ \\
\hline $0-10$ & 55 & 61 & 116 & 11 & 4 & 15 & 12,9 \\
\hline $11-20$ & 48 & 49 & 97 & 10 & 7 & 17 & 17,5 \\
\hline $21-30$ & 21 & 16 & 37 & 4 & 5 & 9 & 24,3 \\
\hline $31-40$ & 34 & 18 & 52 & 11 & 6 & 17 & 32,7 \\
\hline $41-50$ & 14 & 17 & 31 & 7 & 5 & 12 & 38,7 \\
\hline $51-60$ & 14 & 13 & 27 & 6 & 3 & 9 & 33,3 \\
\hline $61-70$ & 5 & 13 & 18 & 2 & 5 & 7 & 38,9 \\
\hline $71-90$ & 6 & 4 & 10 & 4 & 1 & 5 & 50,0 \\
\hline Total & 197 & 191 & $388 \mathrm{~s}$ & 55 & 36 & 91 & 23.4 \\
\hline
\end{tabular}

infectados se alcanzó entre los 31-40 años y luego se incrementó en forma moderada hasta el último grupo de edad considerado.

En lo referente a la tasa de infección discriminada según sexo, se observó predominio en el sexo femenino: $27,4 \%,(55 / 197)$ respecto del masculino: 18,8\%, (36/191); registrándose diferencias levemente significativas $\left(\chi^{2}=3.99\right.$; $\mathrm{p}=0,045)$.

La seroprevalencia de los parajes San Antonio $(26,6 \%)$ y Caimán $(21,4 \%)$ no difirió significativamente $\left(\chi^{2}=1,43 ; \mathrm{p}=0,23\right)$.

Se comprobó además que la presencia de seropositivos en las viviendas no está condicionada a la presencia de perros $\left(\chi^{2}=0,574\right.$; $\mathrm{p}=0,449)$, ni a la de gatos $\left(\chi^{2}=0,677\right.$; $p=0,411)$ que habitan en las mismas.

\section{DISCUSION}

El paisaje general de San Miguel se caracteriza por las escasas posibilidades de trabajo y educación, mínima producción rural, utilizada en forma de subsistencia y traducida en insuficiente ingreso familiar y constante migración a otras regiones con mayores ofertas laborales. Este fenómeno fue verificado en pobladores de edad intermedia quienes vivían en el área de estudio en bajo porcentaje, en coincidencia con lo observado en Córdoba, Argentina ${ }^{18}$. La actual situación epidemiológica de las colonias estudiadas en San Miguel está estrechamente relacionada a las bajas condiciones socio-culturales de los habitantes, entre las que aparecen la precaria vivienda rural, así como las permanentes agresiones ambientales, físicas y biológicas a que están expuestos los pobladores, factores ya señalados para diferentes regiones de América Latina en general y para Brasil en particular ${ }^{10}$.

En este trabajo se confirmó la colonización domiciliaria mayoritaria de $T$. infestans con respecto a $T$. sordida. La presencia de ésta última en una vivienda podría tener origen en la invasión desde biótopos selváticos representados por nidos de Furnariidae, localizados entre 800 y 1.000 metros de la morada.

La ausencia de triatominos en anexos peridomiciliarios podría explicarse por el hecho de que el domicilio por sus características estructurales, continúa siendo el hábitat más favorable para el desarrollo del vector. Asimismo ecótopos silvestres, como palmeras situadas a distancias de solamente 50 metros de las viviendas, ofrecen refugio más propicio para los triatominos, que las estructuras poco protegidas de los peridomicilios.

El elevado porcentaje de perros, gatos y aves que conviven con el morador en la vivienda representan un importante recurso alimentario complementario para mantener a la población de triatominos, ya que se constató que en los domicilios con animales domésticos en su interior fue mayor el número de triatominos vivos colectados.

Más de la mitad de las viviendas que habían sido fumigadas por el Servicio de Chagas y/o Agentes de Salud Rural en las décadas de 1980 y 1990 estaban reinfestadas. La causa de la recolonización sería la carencia de un programa de vigilancia epidemiológica, hecho que debería tenerse en especial consideración, ya que se demostró que se reduce la densidad del vector mediante programas sistemáticos como los efectuados en Río Hondo, Santiago del Estero, Argentina ${ }^{9}$ y en Mambaí, Goiás, Brasil ${ }^{15}$, entre otros.

El porcentaje de infestación doméstica por triatominos en San Miguel es alto, al igual que en otros Dptos. de la provincia de Corrientes, como San Luis del Palmar ${ }^{3}$ e Ituzaingó ${ }^{\circ}$. 
Bar ME, Oscherov EB, Damborsky MP, Alvarez BM, Mizdraji G, Avalos G, Porcel E. Infestacion domestica por Triatoma infestans y prevalencia de seropositivos al Trypanosoma cruzi en un area rural del nordeste argentino. Revista da Sociedade Brasileira de Medicina Tropical 29:549-555, nov-dez, 1996.

La única especie que se encontró parasitada por $T$. cruzi fue $T$. infestans, reafirmando asi su rol como vector principal en Argentina. Además se halló una relación significativa entre la densidad doméstica de $T$. infestans y la densidad de $T$. infestans infectada por $T$. cruzi, en concordancia con lo verificado por Gürtler e cols'ㄹ. En cuanto a la tasa de infección de esta especie en la provincia de Corrientes, resultó similar a la detectada en San Luis del Palmar $r^{3}$ y menor que la de Ituzaingó ${ }^{6}$.

En las localidades estudiadas el 50,0\% de la seroprevalencia se alcanzó a edades menores que en San Luis del Palmar', por lo que el riesgo de transmisión del $T$. cruzi sería mayor en el Dpto. San Miguel. Se resalta además que en más de la mitad de las viviendas infestadas habitaba al menos un seropositivo al $T$. cruzi, lo cual sugiere una activa transmisión del parásito en la población analizada. La prevalencia general obtenida en el presente trabajo está en el orden de la hallada en las proximidades de Resistencia (Chaco) ${ }^{16}$, zona cercana al área de estudio y es menor que la obtenida en Ituzaingó $\sigma^{\circ}$.

El intervalo de edad de mayor riesgo de transmisión del T. cruzi, obtuvo un valor importante en la seroprevalencia y mayor que el registrado en San Luis del Palmar ${ }^{3}$.

Por todo lo antes expuesto se concluye que las condiciones socio-económicas y culturales, entre las que puede citarse la transitoriedad en que se desenvuelve la vida de los pobladores, aparecerían como las responsables del mantenimiento de la endemia en el Dpto. San Miguel.

\section{AGRADECIMIENTOS}

Al Director del Hospital de San Miguel: Dr. P. Ortiz y a los Agentes de Salud Rural por la permanente colaboración prestada a los Dres. C. Wisnivesky-Colli y R. Gürtler por la lectura crítica del manuscrito. Al Banco de Sangre de Corrientes y a los Bioquímicos G. Botello y M.H. Alegre por el apoyo técnico brindado.

\section{SUMMARY}

An epidemiological study was carried out from April 1991 to December 1993 to obtain a general view of $\mathrm{T}$. cruzi domestic transmission in rural areas of San Miguel Department, Corrientes. From 100 analized bousebolds, $50.0 \%$ was infested by $\mathrm{T}$. infestans (Klug, 1834) and 1.0\% by T. sordida (Stảl,
1859). Domiciliary colonization by T. sordida is reported for first time in Corrientes province. $\mathrm{T}$. cruzi infection of $\mathrm{T}$. infestans was $23.1 \%$. Indirect hemagglutination and indirect immunofluorescence antibody tests were used for detection of anti $\mathrm{T}$. cruzi antibodies in 388 buman sera, 23.4\% sbowed serological reactivity. An important bigh rate (12.9\%) was observed in the age group of bigher transmission risk. Seropositives percentages increased with age and reacbed 50.0\% in 31-40 years old group. Domestic infestation by $\mathrm{T}$. infestans, seropositive human pretalence to $\mathrm{T}$. cruzi and bousebolders precarious life conditions prove that this endemic disease is still a problem in the studied area.

Key-words: Triatomines, Infestation. T. cruzi. Seroprevalence. Comientes.

\section{REFERÊNCIAS BIBLIOGRÁFICAS}

1. Alvarez M, Cerisola JA, Rohwedder RW. Test de inmunofluorescencia para el diagnóstico de la Enfermedad de Chagas. Boletín Chileno de Parasitología 23: 4-9, 1968.

2. Bar ME, Oscherov EB. Triatomismo domiciliario de la Provincia de Corrientes, Argentina. Il. Areas rurales del Departamento de Mercedes. Facena 6: 57-66, 1985/86.

3. Bar ME, Oscherov EB, Damborsky MP, Varela ME, Mizdraji G, Porcel E. Triatomismo del Departamento San Luis del Palmar de la Provincia de Corrientes, Argentina. Medicina (Bs. As.) 52: 193-201, 1992.

4. Bar ME, Damborsky MP, Oscherov EB, Alvarez BM, Mizdraji G, Avalos G. Infestación domiciliaria por triatominos y seroprevalencia humana en el Departamento Empedrado, Corrientes,Argentina. Cadernos de Saúde Pública 12(4), 1996.

5. Bejarano JRF, Melega A. Resultado de un muestreo con la prueba de fijación de complemento con antígenos de cultivo de $T$. (S.) cruzi practicado en soldados. In: Primeras Jornadas Entomoepidemiológicas, Bs. As., Argentina. $1^{\circ}$ Parte: p. 405-408, 1959.

6. Borda CE, Rea MJF. Seroepidemiología de la enfermedad de Chagas. Boletín de la Oficina Sanitaria Panamericana 90 :311-323, 1981.

7. Bruniard ED. Bases fisiogeográficas para una división regional de la provincia de Corrientes. Nordeste 8: 7, 1966.

8. Carcavallo RU. Sinopsis epidemiológica de la enfermedad de Chagas. In: Carcavallo RU, Rabinovich JE, Tonn RJ (eds) Factores biológicos 
y ecológicos en la enfermedad de Chagas. OPS. Servicio Nacional de Chagas, Argentina, p. 19-20, 1985.

9. Chuit R. Control vectorial de la enfermedad de Chagas. In: Madoery RJ, Madoery C, Cámera MY (eds) Actualizaciones en la enfermedad de Chagas, Córdoba, Argentina p.265-268, 1993.

10. Dias JCP.Aspectos socio-culturales y económicos relativos al vector de la enfermedad de Chagas. Ini: Carcavallo RU, Rabinovich JE, Tonn RJ (eds) Factores biológicos y ecológicos en la enfermedad de Chagas. OPS. Servicio Nacional de Chagas, Argentina p. 289-304, 1985.

11. Duarte LA. Lineamientos para las políticas de desarrollo urbano de la provincia de Corrientes. Lám.11. Secretaría de Estado de Desarrollo Urbano y Vivienda II. Corrientes. Argentina, 1978/1980.

12. Gürtler RE, Cecere MC, Rubel DN, Schweigmann NJ. Determinants of the domiciliary density of $T$. infestans, vector of Chagas disease. Medical and Veterinary Entomology 6:75-83, 1992.

13 - Lansetti JC, Giordano AD, Subias S, Segura EL. Reacciones serológicas de descarte de la infección chagásica. Medicina (Bs.As.) 40 (Supl. 1): 258-259, 1980 .

14. Lent H, Wigodzinsky P. Revision of the Triatominae (Hemiptera, Reduviidae), and their significance as vector of Chagas' disease. Bulletin of the American Museum of Natural History 163: 123-520, 1979.

15. Marsden P, García-Zapata MT, Castillo EAS, Prata $A R$, Macedo VO. Los 13 primeros años del control de la enfermedad de Chagas en Mambaí, Goiás, Brasil, 1980-1992.Boletín de la Oficina Sanitaria Panamericana 116:111-117,1994.

16. Mendivil GT, Finkielman S, Gorodner JO, Bustamante A, Guilleron CD, Tercelan R, Gatti G, González R. Evidencia epidemiológica de enfermedad cardíaca no relacionada con el $T$. cruzi en zonas endémicas para la enfermedad de Chagas, Medicina (Bs.As.) 44: 489-494, 1984.

17. Mujica LP, Fontana LE, Russo MC, Alvarez M. Encuesta serológica y electrocardiográfica en la población del Departamento de Monte Caseros, Corrientes. Medicina (Bs. As.) 40 (supl.1): 251252,1980 .

18. Ruiz AM, Wisnivesky-Colli C, Gürtler R, Lazzari J, Bujas MA, Segura EL. Infección por Trypanosoma cruzi en humanos, perros y cabras en áreas rurales de la provincia de Córdoba. Medicina (Bs. As.) 45: 539-546, 1985.

19. Schofield CJ. Triatominac. Biología y Control. Eurocommunica Publications, 1994.

20. Siegel S. Estadística no paramétrica. Editorial Trillas, México, 1983.

21. Subsecretaria de Planejamiento. Dirección de Planejamiento. Caracterización socio-económica del Departamento San Miguel. Provincia de Corrientes.Argentina, 1993.

22. Thrusfield M. Epidemiología Veterinaria. Ed. Acribia, SA, Zaragoza, España, 1990.

23. Wisniveski-Colli C, Paulone I, Pérez A, Chuit R, Gualtieri J, Solarz N, Smith A, Segura E. A new tool for continuous detection of the presence of triatominae bugs, vectors of Chagas'disease in rural households. Medicina (Bs. As.) 47: 45- 50, 1987.

24. Wisnivesky-Colli C, Ruiz AM, Gürtler RE, Solarz N, Lazzari J, Ledesma O, Bujas MA, Rissio AM, Marteleur A, Segura E. Dynamics of transmission of Trypanosoma cruzi in a rural area of Argentina. IV. Serologic, parasitologic and electrocardiographic study of the human population. Medicina (Bs.As.) 49:341-350, 1989. 